\title{
Los Estudios de Traducción desde una perspectiva sociológica o la "caja de herramientas" bourdieusiana
}

\section{Laura FÓLICA}

Universitat Pompeu Fabra de Barcelona

España

Resumen: Este artículo se propone estudiar las aportaciones de la sociología de Pierre Bourdieu a los Estudios de Traducción. Para ello, revisaremos las nociones teóricas que elabora Bourdieu, tanto en su sociología del conocimiento como en su teoría social, respecto a la reflexividad del investigador sobre sí mismo y sobre su objeto de estudio. Luego, pondremos a prueba estas nociones en una investigación específica sobre traducción realizada por el traductólogo canadiense J ean-Marc Gouanvic, quien analiza la recepción de la ciencia ficción en la Francia de posguerra.

Palabras clave: Sociología de la Traducción, Recepción literaria, Ciencia ficción, Pierre Bourdieu, J ean-Marc Gouanvic.

Abstract: This article approaches Pierre Bourdieu' sociology and his contributions to Translation Studies. We will look at some theoretical notions developed by Bourdieu in his sociology of knowledge as well as at his social theory regarding the researcher's reflexivity towards himself and his object. Then we will put these notions to test in a specific research about translation developed by the Canadian traductologist J ean-Marc Goaunvic, who analyses the reception of science-fiction in postwar France.

Keywords: Translation Sociology, Literary Reception, Science-fiction, Pierre Bourdieu, J ean-Marc Gouanvic.

El objetivo del presente artículo es estudiar las aportaciones de la sociología de Pierre Bourdieu (1930-2002) a los estudios de traducción. Si bien, en su extensa obra, Bourdieu no reflexionó sobre la traducción ${ }^{1}$ como práctica social ni la consideró como objeto de estudio,

1 Como excepción, puede mencionarse el diálogo entre Bourdieu y uno de sus traductores, Richard Nice, en el libro Traduire l'Europe (volumen dirigido por Françoise Barret-Ducrocq, 1992) o la referencia secundaria a los traductores en un 
los conceptos por él elaborados para comprender y explicar el funcionamiento del campo del arte, los medios y la edición resultan productivos en el ámbito traductológico. De este modo, haciendo de la teoría una "caja de herramientas" -tal como sugerían Deleuze y Foucault $-{ }^{2}$ nuestro ejercicio será medir dicha productividad en una investigación pionera sobre la sociotraducción, realizada por el investigador canadiense Jean-Marc Gouanvic, quien analiza la importación de la ciencia ficción norteamericana en el espacio cultural francés de los años 1950.

Así pues, rastrear en una investigación concreta sobre traducción nociones teóricas de la sociología bourdieusiana es el objetivo que animará el artículo, conforme con el principio de imbricación entre teoría, práctica y metodología propuesto por el propio Bourdieu. En ese sentido, haremos algunas aclaraciones, en el primer apartado, sobre el Bourdieu epistemólogo que elabora una sociología del conocimiento: estudiaremos las rupturas con cierto sentido común sobre lo social que debe realizar el investigador, así como el ejercicio de reflexividad sobre su posición como analista. Luego veremos cómo es asumida esta reflexividad en la investigación elegida de Gouanvic.

En el segundo apartado, revisaremos la sociología de bienes simbólicos de Bourdieu, es decir, su sociología orientada a los productos artísticos; estos objetos se caracterizan por poseer un valor no solo económico sino también, y sobre todo, simbólico, operando como marca de distinción del poseedor. A continuación, veremos cómo las nociones centrales de dicha sociología son asumidas en la investigación de Gouanvic. Es dable aclarar que ambas sociologías de Bourdieu, esto es, tanto sus reflexiones sobre el papel de investigador como sus formulaciones teóricas sobre el arte, se exponen en investigaciones empíricas situadas en un espacio y tiempo determinados (la mayor parte, en la Francia contemporánea) en las que el autor desarrolla sus conceptos en relación con los objetos que estudia; de ahí que sea difícil aislar una única definición de sus nociones más conocidas.

\subsection{La reflexividad sobre el investigador: la sociología del conocimiento de Bourdieu}

Según Bourdieu, el conocimiento científico en las ciencias sociales avanza por medio de una lógica de rectificación permanente en la propia práctica de la investigación. Alejándose de la mera

artículo sobre el mundo de la edición "Une révolution conservatrice de l'édition" (Bourdieu 1999).

2 Deleuze afirma en una entrevista junto a Foucault $(1972,5)$ : “C'est ça, une théorie, c'est exactement comme une boîte à outils. [...] Il faut que ça serve, il faut que ça fonctionne. Et pas pour soi-même. S'il n'y a pas des gens pour s'en servir, à commencer par le théoricien lui-même qui cesse alors d'être un théoricien, c'est qu'elle ne vaut rien, ou que le moment n'est pas venu”. 
comprobación científica del hiperempirismo que ubicaría al objeto como un hecho dado y dispuesto a ser observado, el autor realiza una primer ruptura, "la ruptura con lo real" (Bourdieu et al 1975, 27-50), que significa oponerse a la reproducción del sentido común más propio de una sociología espontánea. Así pues, Bourdieu plantea la construcción relacional del objeto de investigación para las ciencias sociales: no se trata de emular a las ciencias naturales e ir en busca del objeto en "el mundo real" y "recortarlo" sin injerencia del observador; todo lo contrario, el objeto del analista será la construcción que él mismo elabore como problema a resolver en su investigación.

En otros términos, para Bourdieu, el objeto de investigación ha de conquistarse (el analista debe romper con las relaciones más aparentes y familiares del mundo social), ha de construirse (como hemos dicho antes, el analista no puede equiparar el objeto real con el objeto de estudio) y ha de comprobarse (el analista buscará hacer ciencia y no una sociología de carácter especulativo).

Una segunda ruptura que realiza Bourdieu es contra el metodologismo y el teoricismo. El primero es practicado por investigadores que desarrollan una fidelidad a ultranza hacia la técnica de investigación; el segundo, en cambio, deja de lado la forma de medir los datos y se centra en la reflexión teórica; en ambos casos, método y objeto se hallarían disociados, imponiéndose o bien una forma de medición o bien un abordaje teórico específico que desatiende las particularidades del objeto. De ahí que Bourdieu prefiere romper con el "rigor sin imaginación del positivismo hiperempirista" y con la "audacia sin rigor de la filosofía social” (Bourdieu y Wacquant 1995, 31) y seguir el camino de lo que denomina "relacionismo metodológico" (23). Evitando caer en un exceso de cautela metodológica, el analista tendrá más libertad en elegir, entonces, el método y las técnicas más convenientes para la construcción de su objeto en relación con su problema específico. También se revaloriza el aspecto práctico de la teoría como herramienta productora de conocimiento. A modo de ejemplo, podemos ver que el mismo Bourdieu utiliza diferentes materiales en sus investigaciones de acuerdo con las preguntas que se formula. Así, en su libro Las reglas del arte, el análisis de una novela de Flaubert le permitirá estudiar la génesis del campo literario francés, en tanto que en su obra La distinción, al interrogarse sobre el consumo de las obras de arte, se servirá de encuestas y de entrevistas en profundidad a los consumidores franceses.

Por último, dado que la perspectiva es constructiva y relacional, el lugar ocupado por el investigador será otra ruptura. Bourdieu polemiza con el hiperempirismo que no problematiza la posición del observador, al reducirlo a mero corroborador de un objeto externo. Para nuestro autor, en cambio, el privilegio del sociólogo consiste en reflexionar sobre su propia posición en el campo, esto es, objetivar su posición para evitar caer en la reproducción de los prejuicios propia de una sociología espontánea. Así es que propone que el investigador realice un 
socioanálisis, práctica que nada tiene que ver con una perspectiva psicológica o empática. Se trata más bien de que el investigador reflexione sobre las determinaciones de lo social que lo atraviesan como sujeto $\mathrm{y}$, en este caso, como analista. Además, el ejercicio del socioanálisis reviste una dimensión ética de grupo si se extiende su práctica a todos integrantes del campo científico. Dicho de otro modo, si se institucionaliza el socioanálisis como práctica reflexiva de los investigadores, las ciencias sociales apuntarán a una mayor honestidad intelectual al revisar sus posiciones y vigilar sus herramientas teóricas.

\subsection{La reflexividad sobre el analista en una investigación específica de traducción}

En el libro Sociologie de la traduction. La Science-Fiction américaine dans l'espace culturel français des années 1950, el traductólogo canadiense Jean-Marc Gouanvic comparte la postura epistemológica de Bourdieu y su gesto de ruptura en contra de una sociología espontánea. De esta manera, podemos pensar que Gouanvic rompe con "lo real", pues no estudia meramente la ciencia ficción como un dato externo a describir, sino que construye su objeto de análisis: la recepción del género de ciencia ficción en el espacio cultural francés de posguerra. Además, como veremos en el siguiente apartado, evita caer en el metodologicismo al emplear un método de análisis no ortodoxo adecuado a su objeto. Así reúne, por un lado, el análisis del discurso y, por otro, el estudio descriptivo del aparato importador de ciencia ficción (tomando en consideración a editoriales, colecciones, fanzines que intervinieron en la introducción del género). Y sobre todo, la adscripción bourdieusiana se hace evidente antes de comenzar el estudio de su objeto, al plantear la necesidad de realizar la revisión de su posición como investigador, esto es, su propio socioanálisis, aplicando al traductólogo aquello que Bourdieu pretendía para el sociólogo:

De même que le sociologue selon Bourdieu ne peut s'exclure du jeu social qui est l'objet de son analyse, de même le traductologue ne peut se mettre «hors-jeu » dans son étude des phénomènes traductifs (Gouanvic 1999, 144).

Si así lo hiciera, perdería el rico componente social de su reflexión. Con tal propósito, Gouanvic dedica la introducción y la conclusión de su libro a aclarar su posición como traductólogo en el campo académico. ${ }^{3}$

Si bien afirma que "nous ne présenterons pas ici un état actuel des recherches en traductologie, nous en tenant aux considérations susceptibles d'éclairer notre propos" (13), el autor perfila un posible

\footnotetext{
3 Gesto que prolongará, aunque más interesado en abordar la ética en la traducción incorporando la lectura de Antoine Berman y Henri Meschonnic, en su siguiente libro: Pratique sociale de la traduction - Le roman réaliste américain dans le champ littéraire français (1920-1960).
} 
"estado del arte" de las investigaciones en traducción, para luego inscribir su estudio no en continuidad o "mejora" de ciertas teorías traductológicas, sino en el marco de una sociología general de la traducción.

Gouanvic propone diferentes ordenamientos, y esto nos permite advertir el carácter construido de toda clasificación:

Les traductologues sont des agents qui luttent pour imposer les classements théoriques de la discipline et, par là même, la problématique légitime dans le champ de la traductologie (15).

Al afirmar esto, el autor hace patente que las clasificaciones no son estáticas, sino que cobran dinamismo y, por lo tanto, pueden modificarse. 4

Según Gouanvic, un posible ordenamiento de los estudios de traducción sería distinguir entre quienes se ocupan del texto/la lengua/la cultura fuente (por ejemplo, la poética de la traducción de Meschonnic) y quienes se ocupan del texto/la lengua/la cultura meta (por ejemplo, la teoría del polisistema de Even-Zohar). Otro ordenamiento podría ser el que reúne los estudios según el modo de construcción del objeto. En ese sentido, el autor señala que desde 1970, algunos análisis se centraron en el "proceso" (el cognitivismo en Finlandia y Alemania), otros en el "producto" (la traducción literaria estudiada por André Lefevere), y otros en la "función" (los estudios de traducción descriptivos desarrollados en Israel y Bélgica).

Gouanvic advierte que una década después, en 1980, comienza a problematizarse la idea de lo social "inscripto" en el texto traducido. Esta perspectiva será compartida por estudios de cariz más cultural: la teoría del polisistema (Toury 1995), la sociocrítica de los textos (Brisset 1990) y la teoría escópica (Nord 1993). El autor reconoce la preocupación por lo social en las aportaciones de los autores mencionados, quienes focalizan, desde diferentes ángulos, en la traducción como fenómeno que integra la recepción de una obra. Ahora bien, Gouanvic considera que aún faltaba desarrollar una "sociología de las producciones simbólicas" que aplicara el pensamiento de Bourdieu en el estudio de la traducción, analizando el lugar del traductor como agente, su posición en el campo y su habitus (nociones que abordaremos en el apartado siguiente). Y es allí, justamente, donde inscribirá la pertinencia de su investigación. ${ }^{5}$

\footnotetext{
4 En ese sentido, P. Bourdieu y L. Wacquant $(1995,20)$ declaran la utilidad de revisar las clasificaciones naturalizadas. Estas debieran ser consideradas como productos sociales, construcciones con una función política al ser un instrumento de dominación de los sistemas simbólicos que se naturalizan.

5 En ese sentido, Hélène Buzelin $(2005,203)$ en un volumen de The Translator dedicado a las aportaciones de la sociología para la traducción destaca: "Jean-Marc Gouanvic recently presented his attempt at 'adapting Bourdieu's theory to translation studies' as a way of integrating the agent into the analysis, an element which, to him had 'absolutely no role, nor place in polysystemic studies".
} 
Entonces, si bien el autor reconoce que las investigaciones teóricas en traducción nacieron de la mano de la lingüística, no considera que la existencia del campo de los estudios de traducción deba ubicarse necesariamente en continuidad con ellas. Existiría, pues, una ruptura entre esos lingüistas pioneros que estudiaron la traducción (Jakobson, Nida, Vinay y Darbelnet, Mounin, Catford) y los traductólogos actuales, que se interesan por la dinámica del campo específico de los estudios de traducción. En efecto, podemos pensar que la reflexión teórica sobre la traducción se modifica cuando se conforma un campo específico, con estructuras, reglas y apuestas propias y con agentes que poseen un interés material y/o simbólico en la toma de posición sobre la traducción. Este campo se va construyendo a partir de nuevas estructuras institucionales favorables a su consolidación: la fundación de asociaciones de traductores, la creación de revistas académicas, la convocatoria a reuniones científicas y la oferta académica formativa y de investigación en el área. Además, Gouanvic va más allá y plantea una revisión general de las herramientas conceptuales. Así, para evitar caer en una "traductologie demeurant le parent pauvre des sciences humaines" (147), los traductólogos deberían revisar nociones como fidelidad, equivalencia, función y problematizar la práctica de la traducción y su relación con el conjunto de prácticas socioculturales en las que ella interviene.

Hasta aquí, nos hemos referido a la reflexión epistémica del traductólogo, necesaria para elaborar el punto de vista sobre su objeto. A continuación, abordaremos la sociología de los bienes simbólicos de Bourdieu para, luego, ver cómo funciona en la investigación del propio Gouanvic.

\subsection{La reflexividad sobre el objeto de investigación: la sociología de los bienes simbólicos de Bourdieu}

Dejando de lado los ejes antropológico y educativo presentes en los trabajos de Bourdieu, el aspecto de su teoría social que nos interesa es su sociología del arte o su sociología de los bienes simbólicos.

Ahora bien, ¿por qué es necesaria dicha sociología? Para Bourdieu, la creación artística (o literaria) puede y debe ser explicada desde una perspectiva sociológica. La explicación radica en que, por un lado, este enfoque permite ir contra una lectura de tipo interna, que focaliza en la obra como una creación única; tal postura hermenéutica acaba por deshistorizar la obra, alejándola de su contexto. Pero, por otro lado, tampoco se debería caer en una lectura externa que explique la obra por la concepción psicologista o de clase del autor (en este punto, por ejemplo, Bourdieu discute con Sartre, quien en L'Idiot de la famillie caracteriza a Flaubert según su posición en el seno de su familia).

Bourdieu propondrá, en cambio, una lectura relacional del autor con su obra y con el campo literario. Este abordaje resultaría superador de dos comportamientos en los que a veces cae el investigador: el 
objetivismo (en el que se acaba privilegiando la estructura por sobre los sujetos) y el subjetivismo (en el que, al contrario, se rescata la acción individual del sujeto sin tener en cuenta la posición que ocupa dentro de una estructura). En ese sentido, Bourdieu se define a medio camino como "constructivismo estructuralista"; así pues, podemos pensar que tanto el objetivismo como el subjetivismo no se excluyen, sino que resultan dos instancias necesarias en una investigación.

En su modelo, marcado fuertemente por las metáforas espaciales, Bourdieu prefiere referirse a la sociedad en términos de "espacio social", el cual está conformado por diferentes "campos". Si bien el autor va componiendo distintas definiciones de sus conceptos, podríamos caracterizar al campo como un espacio relativamente autónomo de relaciones histórico-objetivas entre los agentes, que ocupan distintas posiciones de poder.

Aunque cada campo (cualquiera sea, económico, escolar, artístico, etc.) goza de relativa autonomía, existen mecanismos o leyes generales de funcionamiento (Bourdieu 2002, 119-126): todo campo presenta una parte dominante y una dominada, un capital puesto en juego (económico, escolar, literario, etc.) y agentes dispuestos a luchar por mantener o modificar la posesión de su capital. Cabe aclarar que si bien los agentes compiten, ellos comparten cierta complicidad al aceptar el juego, al tiempo que ayudan a la reproducción del mismo.

Una vez trazado el espacio objetivo del campo, Bourdieu agrega una segunda instancia en el análisis: el momento subjetivo donde introduce la propia percepción de los sujetos en la estructura, sus "disposiciones o esquemas de percepción, pensamiento y acción" (Bourdieu 2002, 49), que el autor denomina habitus.

\subsection{La reflexividad sobre el objeto en una investigación específica de traducción}

Si bien, como hemos dicho, Bourdieu no estudió especialmente la traducción, su mirada resulta productiva para pensar la producción, circulación y consumo de textos extranjeros traducidos. Así pues, respecto de la traducción como práctica cultural, creemos que esta puede inscribirse en el "campo intelectual", justamente aquel en el que se producen los bienes simbólicos. En este campo, si bien los otros capitales están presentes, la importancia de lo simbólico es clave puesto que la lógica específica de este campo es la de la competencia por la "legitimidad cultural" (Altamirano y Sarlo 1990, 15). En este caso, los agentes legitimadores (por ejemplo, los editores, los críticos, los periodistas culturales, etc.) desarrollan estrategias de selección y de consagración, decidiendo sobre las normas de traducción, los autores a traducir, la difusión de los libros, etc. De ahí que Bourdieu señale que se trata de un malentendido pensar que "los textos circulan sin sus contextos" (Bourdieu 2002b, 4); desde esta perspectiva sociológica, en todo análisis de una traducción debiera considerarse no solo la obra, 
sino el campo de partida y el de llegada y, en este último caso, la relación con obras de producción local y con sus estructuras institucionales. Para caracterizar dicha relación -y recuperando aquí la teoría del polisistema (Even-Zohar 1979)- pensamos que la traducción como modo de introducir literatura extranjera puede desempeñar dos papeles opuestos: o bien conservador (es decir, reforzar los modelos ya existentes en la cultura de llegada) o bien innovador (esto es, alterar el modelo con géneros nuevos).

Yendo a la investigación sobre la traducción de ciencia ficción que analizamos, notamos que Gouanvic se sirve de la sociología de los bienes simbólicos para reflexionar sobre la traducción. El autor señala que si bien se ha estudiado a la ciencia ficción como género, no ha ocurrido lo mismo con su traducción, que permaneció como el "punto ciego" en el análisis científico. Por eso es que resulta útil tomarla como objeto de estudio no solo porque la traducción evidencia la circulación de las lenguas, la presencia de lo extranjero, la innovación, sino también porque muestra

[...] les transformations que subissent nécessairement dans et par le processus de la traduction les productions d'une culture (culture source) importées dans une autre culture (culture cible) à un moment de son histoire, mais encore les positions de pouvoir assumées par les traductions et leurs agents dans leurs champs spécifiques. (Gouanvic 1999, 11)

Gouanvic analiza la importación masiva de un tipo de texto extranjero: la traducción de novelas y relatos de un género literario nuevo, la ciencia ficción norteamericana, en un espacio cultural (la Francia de posguerra), que estuvo acompañada de una importación de estructuras institucionales extranjeras (colecciones, revistas, fans clubs, premios, etc.). En otras palabras, desde una concepción ampliada de traducción, su objetivo es analizar los fenómenos culturales que hacen que los textos extranjeros existan traducidos en la sociedad meta, integrados al campo intelectual receptor.

El libro comienza con una explicación sobre la traducción del sintagma "ciencia ficción". Esta aclaración que, en un principio, podría pensarse marginal, ilustra, sin embargo, de modo metonímico la estrategia imperante de importación de este género en Francia. La traducción del término en francés es science-fiction, es decir, un calco de estructura (Vinay y Darbelnet 1958, 47) cuya formación no se corresponde con la sintaxis francesa ordinaria (en la que se hubiera preferido: fiction de science, fiction-science, fiction scientifique). Esta elección no es menor, ya que genera un efecto de reconocimiento del origen extranjero (norteamericano) del género. En efecto, el calco del sintagma caracteriza el tipo de relación entre la cultura francesa y la cultura norteamericana. La ciencia ficción se habría introducido en Francia como una literatura innovadora, en ruptura con los textos y géneros locales; la búsqueda, entonces, no era la de la aclimatación, 
sino, todo lo contrario, la de la marcación de la extranjeridad de esa literatura, es decir, que se tratara de una literatura foránea.

Para llegar al momento de éxito de introducción de la ciencia ficción, que se vio acompañada de una estrategia editorial mayor, Gouanvic caracteriza el campo literario de partida de los Estados Unidos en 1920, fecha de aparición y homogeneización del género de ciencia ficción y momento en el que se estabiliza el nombre del mismo género. La ciencia ficción va afianzándose a medida que se conforma el campo específico con modelos institucionales, subcultura, sociolectos, gustos, costumbres, etc.

La importación de este género al campo literario francés se dio con éxito en 1950, pero hubo intentos anteriores, más aislados, como la recepción de las traducciones francesas de $\mathrm{H}$. G. Wells en instituciones prestigiosas como el Mercure de France y la $N R F$, a comienzos del siglo $\mathrm{XX}$.

Gouanvic afirma que los primeros intentos para implantar la ciencia ficción norteamericana en Francia por los años 1930 estuvieron destinados al fracaso. La estrategia de selección se había centrado en la importación de autores "competentes" en el campo fuente (Régis Messac y Georges-Hilaire Gallet), pero importados aisladamente. A continuación, el autor repasa los intentos exitosos de importación durante la posguerra. En este caso, entonces, el éxito se debió a que la traducción de la obra estuvo acompañada por modelos editoriales y discursivos, es decir, se concibió a la traducción de un modo ampliado al incorporar no solo las estrategias de traducción, sino también las editoriales. A propósito de esto, si bien Gouanvic no lo considera porque su libro es anterior, creemos útil mencionar aquí el artículo de Bourdieu "Les conditions sociales de la circulation internationale des idées" (2002b), en el que se afirma que la transferencia de una obra se realiza conforme con operaciones sociales de selección, marcación y lectura.

En el caso de la ciencia ficción, la selección de los textos se realizó en base a las obras consagradas en la cultura fuente; pero a condición de que esta consagración fuese reconocida como legítima por la sociedad meta. Dicho reconocimiento solo es obtenido si la legitimada del texto sirve a los intereses de los agentes del espacio cultural meta. En este caso, las obras fueron importadas por agentes legitimadores, esto es, agentes que gozaban de prestigio previo en el campo de llegada y que, en sus discursos, legitimaban la implantación del género en el espacio cultural francés. Se trataba de escritores como B. Vian, R. Queneau y M. Pilotin. La segunda operación de transferencia citada por Bourdieu es la marcación. Esta se lleva adelante en prefacios, tapas y colecciones específicas (como, por ejemplo, la creación de la colección "Le Rayon Fantastique"), que orientan la obra al campo de llegada. Por último, la tercera operación es la lectura, no en términos subjetivos, sino siempre mediada por el campo de llegada, que realizan los lectores de ciencia ficción. 
Así como la selección e importación estuvo a cargo de agentes legitimados en la cultura francesa, la estrategia de traducción dominante que emplearon estos traductores fue la de exotización; esta consiste en dejar las marcas de origen del texto de partida, no aclimatándolo a la cultura de llegada, de ahí que se la suela presentar como estrategia opuesta a la domesticación. Marcar el rasgo norteamericano en la traducción gozaba de legitimidad entre el público de entonces, porque en la posguerra Estados Unidos era visto como la cuna del género de ciencia ficción, a tal punto que autores franceses se vieron tentados a escribir ciencia ficción con seudónimos ingleses; mencionamos, por ejemplo, el de Vernon Sullivan (seudónimo polémico de Boris Vian) .

Respecto del método seguido en su investigación, al estudio estructural de las editoriales y colecciones, Gouanvic añade un análisis discursivo. Así pues, para dar cuenta de la estrategia de traducción utilizada, dedica tres capítulos a una comparación microtextual de las traducciones publicadas en la colección "Le Rayon Fantastique", las traducciones de Boris Vian y aquellas realizadas por la revista Galaxia. Este último caso constituye una excepción a la estrategia hegemónica de exotización, ya que, en dicha publicación, la onomástica extranjera fue aclimatada con nombres franceses. Gouanvic arriesga como hipótesis que Galaxia buscó desarrollar una estrategia atractiva, por lo novedoso de la propuesta, en un momento en que la experiencia de lectura del género ya era mayor en el campo francés, dado que la publicación de la revista se extiende hasta 1977.

Por último, para completar la propuesta bourdieusiana, cabe incorporar la instancia de análisis más subjetivo asociada al habitus del traductor. El habitus, siempre asociado a una clase o a un grupo, moldea, pues, las prácticas de los sujetos y sus estrategias. Por ejemplo, el traductor será poseedor de un habitus de grupo que orientará su forma de percibir, de pensar y de actuar en relación con la traducción. En ese sentido, podemos pensar en el célebre tópico de la "invisibilidad del traductor" (Venuti 1995) como un rasgo caracterizador del habitus del traductor (quien suele ver con buenos ojos borrar su enunciación para que la traducción "no se note").

Si bien Gouanvic rescata el concepto de habitus como un punto central y novedoso en la sociología de Bourdieu 6 , no se detiene demasiado en su estudio. A grandes rasgos, rescata cierta homología de habitus de los agentes importadores, esto es, de los escritores norteamericanos con los traductores franceses, ya que ambos estaban interesados en desarrollar un discurso crítico de los valores tradicionales. Gouanvic sugiere que los traductores apelaron, por intermedio de la ciencia ficción, al topos de la protesta y del "género nuevo", una forma de síntesis de dos culturas: la científica y la literaria. Cabe destacar también el clima de época de posguerra compartido por

\footnotetext{
${ }^{6}$ Véase nota 5.
} 
cierta pequeña burguesía tecnófila, la cual adhería al american way of life, que se erigía como un sueño posible luego de la guerra.

\section{Conclusión}

En el presente trabajo nuestro objetivo fue plantear la doble reflexividad -sobre el analista y sobre el objeto de estudio- que caracteriza a la investigación en ciencias sociales. Por un lado y en primera instancia, la reflexión sobre el lugar del investigador en su propio campo académico, que permite obtener una mirada más honesta a la hora de dar cuenta del objeto de investigación elegido. Hemos visto, entonces, que este ejercicio aconsejado por Bourdieu lo lleva adelante el investigador Gouanvic en su propia investigación al construir un estado del arte sobre el campo de los estudios de traducción, donde él mismo se ubica como analista, desarmando las categorías heredadas y haciendo explícita la conformación y consolidación de este campo.

Por otro lado, la reflexividad sobre el objeto nos llevó a explicar la propuesta de la sociología de los bienes simbólicos de Bourdieu. Hemos visto que esta resulta útil para concebir a la traducción como una práctica cultural inserta en una dinámica de circulación entre campos. Dicha circulación, lejos de aparecer como una traslación horizontal, permite poner en evidencia la lucha simbólica por la legitimidad de determinadas selecciones de autores, obras y traductores. Esto lo pudimos corroborar en la investigación de Gouanvic, quien revisa los intentos -unos fallidos, como los de Messac y Gallet, y otros exitosos, como los de Vian, Queneau y Pilotin- de importación de la ciencia ficción en el campo francés de posguerra. Asimismo, revisamos las operaciones de selección y marcación que permitieron la introducción y afianzamiento del género nuevo: en lugar de adaptar a la cultura francesa, la estrategia consistió en mantener el color local del texto de partida, guardando el exotismo norteamericano.

Tras lo expuesto, creemos que la puesta a prueba del modelo bourdieusiano en una investigación pionera de traducción muestra que el modelo sociológico resulta productivo para pensar las prácticas ligadas a la traducción. Este marco teórico nos permite abandonar la subsidiaridad hacia la cultura y lengua fuente, que dominó en el comienzo de los estudios de traducción como disciplina, y pasar a reflexionar sobre los diferentes factores lingüísticos, pero también sociales y económicos, que están presentes en los intercambios culturales. La noción de "campo" remite a una estructura más dinámica y relacional; asimismo, el concepto de "agente" poseedor de un habitus nos lleva a hacer foco en los diferentes perfiles de mediadores que intervienen en la introducción de una literatura: traductores, prologuistas, editores, directores de colección, y en sus prácticas y estrategias de exotización o domesticación.

A partir de este estudio pionero de Gouanvic, durante la primera década del siglo XXI, más investigaciones han recurrido a Bourdieu para 
el análisis de fenómenos asociados con la traducción. Esto demuestra la productividad del modelo y, en ese sentido, permite el control y mejora de los avances traductólogicos en el campo, tal como preveía Bourdieu en su propuesta de socioanálisis. Podemos mencionar algunos ejemplos: desde el propio Gouanvic, quien, en un segundo libro, analizó la importación de la novela realista norteamericana en el campo literario francés entre 1920 y 1960; hasta publicaciones que reúnen varias contribuciones, útiles para conocer a los investigadores que trabajan desde esta óptica. Así pues, el número 145 de la revista Actes de la recherche en sciences sociales de 2002 resume diferentes investigaciones sobre traducción a cargo de autores como Casanova, Shavit, Wilfert, Kalinowski, Popa, Serry, Sapiro y Heilbron. En el mundo anglosajón, por su parte, el número especial de la revista The Translator de 2005 reúne artículos de varios investigadores que buscan vincular a Bourdieu con otros marcos teóricos como la teoría de redes de Latour; citamos a Buzelin, Inghilleri, África Vidal Claramonte, entre otros. En la misma línea de enriquecimiento de los estudios de traducción con otras perspectivas sociológicas, cabe mencionar los volúmenes Why translation studies matters?, de 2010, y Constructing a Sociology of translation, de 2007, en el que se incorporan las aportaciones de otros sociólogos como Lahire, Giddens o Luhmann.

En general, en las investigaciones de análisis de caso que surgieron en este inicio de siglo, notamos que se suele repetir el modelo usado por Gouanvic en la construcción del objeto de análisis: la importación de un autor, tipo de texto o género en un campo cultural en un período de tiempo acotado. Si bien hemos visto que, en Bourdieu, tanto el momento objetivo como el subjetivo eran complementarios para el análisis, advertimos que en las nuevas investigaciones sigue prevaleciendo el análisis de la estructura del campo (excepciones interesantes son el artículo de Kalinovsky (2001) sobre el habitus del traductor literario, así como otro de Simeoni (1998) o el segundo libro del propio Gouanvic).

También cabe destacar que la lógica de lo nacional sigue prevaleciendo a la hora de estudiar los campos, pues se suele acotar el espacio social a los campos nacionales autónomos. Ahora bien, esta perspectiva debe revisarse actualmente a partir de lecturas más internacionales que problematizan la importación-exportación de literatura en términos globales y de interdependencia. En ese sentido, ¿acaso es posible hablar de un campo internacional?, ċo es preferible el empleo de una nueva terminología? Autores como Heilbron, Sapiro, Wolf o Prendergast reflexionan en esa dirección, describiendo las relaciones de poder que intervienen en el flujo de intercambio desigual de libros y demostrando que el modelo bourdieusiano puede enriquecerse desde una reflexión que tenga en cuenta la dinámica de la globalización. 


\section{Referencias bibliográficas}

Altamirano, Carlos, Beatriz Sarlo. Conceptos de Sociología Literaria. Buenos Aires: C.E.A.L., 1990.

Bourdieu, Pierre, Jean-Claude Chamboredon, Jean-Claude Passeron. El oficio del sociólogo. Buenos Aires: Siglo XXI, 1975 [original francés: Le métier de sociologue. París: Mouton, 1983].

Bourdieu, Pierre, Loïc Wacquant. Respuestas para una antropología reflexiva. México: Grijalbo, 1995 [original francés: Réponses pour une anthropologie réflexive. París: Le Seuil, 1992].

Bourdieu, Pierre. "Une révolution conservatrice de l'édition». Actes de la recherche en sciences sociales, 1999, $\mathrm{N}^{\mathrm{O}}$ 126-127: 3-28, Bourdieu (ed.). París: Seuil.

Bourdieu, Pierre. «Algunas propiedades de los campos». Campo de poder, campo intelectual. Buenos Aires: Montressor, 2002. [original francés: «Quelques propiétés des champs». Questions de sociologie. París: Les Éditions de Minuit, 1984].

Bourdieu, Pierre. «Campo intelectual y proyecto creador». In : Campo de poder, campo intelectual. Buenos Aires: Montressor, 2002. [original francés: «Champ intellectuel et projet créateur». Les Temps modernes, 1966, No 246: pp. 865-906].

Bourdieu, Pierre. «Les conditions sociales de la circulation internationale des idées». Actes de la recherche en sciences sociales, $2002 \mathrm{~b}, \mathrm{~N}^{\circ} 145: 3-8$. Bourdieu (ed.). París: Seuil.

Brisset, Annie. Sociocritique de la traduction. La traduction théâtrale au Québec 1968-1988. Longueil: Éditions du Préambule, 1990.

Buzelin, Hélène. «Unexpected Allies: How Latour's Network Theory Could Complement Bourdieusian Analyses in Translation Studies». The Translator, 2005, Vol. 11, $\mathrm{N}^{0}$ 2. Moira Inghilleri (ed.). Manchester: St. Jerome Publishing.

Even-Zohar, Itamar. «Polysystem Theory». Poetics Today, 1979,Vol. 1, No 1-2: pp. 287-310.

Foucault, Michel, Gilles Deleuze. "Les intellectuels et le pouvoir». L'Arc, 49, 1972. Retomado en Michel Foucault. Dits et écrits II. París: Gallimard, 1994, p. 309.

Gouanvic, Jean-Marc. Sociologie de la traduction - La Science Fiction américaine dans l'espace culturel français des années 1950. Arras: Artois Presses Université, 1999.

Gouanvic, Jean-Marc. Pratique sociale de la traduction - Le roman réaliste américain dans le champ littéraire français (1920-1960) Arras: Artois Presses Université, 2007.

Heilbron, Johan. «Toward a Sociology of Translation. Book Translations as a Cultural World-System». European Journal of Social Theory, 1999, Vol. 2, No 4: 429-444.

Nord, Christiane. Einführung in das funktionale Übersetzen. Am Beispiel von Titeln und Überschriften. Tubinga: Francke, 1993.

Kalinovsky, Isabelle. «La vocation au travail de traduction». Actes de la recherche en sciences sociales, 2002, $\mathrm{N}^{\mathrm{O}}$ 145. Gisèle Sapiro y Johan Heilbron (eds.). París: Seuil.

Prendergast, Christopher. «Negotiating world literature». New Left Review, marzo-abril 2001, No 8: 100-121, London.

Sapiro, Giselle. Translatio. Le marché de la traduction en France à l'heure de la mondialisation. París: Éditions du CNRS, 2009. 
Simeoni, Daniel. "The Pivotal Status of the Translator's Habitus». Target, 1995, Vol. 10, No 1: 1-39. Amsterdam-Filadelfia: John Benjamins.

Toury, Gideon. Descriptive Translation Studies and beyond. AmsterdamFiladelfia: John Benjamins, 1995.

Venuti, Lawrence.The Translator's Invisibility: A History of Translation. London: Routledge, 1995.

Vinay, Jean-Paul, Jean Darbelnet. Stylistique comparée du français et de l’anglais. París: Didier, 1958. 